\title{
Giant Magnon in NS5-brane Background
}

\author{
J. Klusoň \\ Dipartimento di Fisica, \\ Universita' 83 I.N.F.N. Sezione di Roma 2, Tor Vergata \\ Via della Ricerca Scientifica 1, 00133 Roma, ITALY \\ E-mail: Josef.Kluson@roma2.infn.it \\ Rashmi R. Nayak \\ Center for Quantum Spacetime (CQUeST), \\ Sagang University, Seoul, 121-742, Korea \\ E-mail: rashmi@sogang.ac.kr
}

\section{Kamal L. Panigrahi}

Department of Physics

Indian Institute of Technology, Guwahati, 781 039, Guwahati, INDIA

E-mail: panigrahi@iitg.ernet.in

\begin{abstract}
We study the giant magnon solutions in the near horizon geometry of the Neveu-Schwarz (NS) 5-brane background. In conformal gauge, we find magnon dispersion relation in the large angular momentum $(J)$ limit. We further show that the giant magnon poses uniform distribution of the angular momentum along the string world-sheet as in case of $A d S_{5} \times S^{5}$ spacetime.
\end{abstract}

KEYwORDs: D-branes. 


\section{Contents}

1. Introduction 1

2. Polyakov string in the near horizon limit of NS5-brane background 3 2.1 Infinite $J$ Giant Magnon 8

3. Uniform light-cone gauge 9

4. Conclusions 15

\section{Introduction}

Type IIB String theory on $A d S_{5} \times S^{5}$ has been conjectured to be dual to $\mathrm{N}=4$ super Yang-Mills theory in 4-dimensions [1]. The conjectured duality has passed through various nontrivial tests in the past by analyzing the spectrum of quantum string states on $A d S_{5} \times S^{5}$ background and the spectrum of the anomalous dimensions of the $\mathrm{N}=4$ gauge theory operators in the planar limit. Especially in the semiclassical approximation the theory become integrable on the both sides of the duality ${ }^{1}$. Though finding out the full spectrum of string theory in AdS background is still an open unsolved problem, it has been observed that in certain sectors of the theory it is more tractable. One of them is the large angular momentum sector of the theory [2]. In this region, one can use the semiclassical approximations to find the string spectrum as well [3]. The correspondence then demands to find out a particular type of operators that are the long trace operators in the gauge theory side. This fact was resolved by an amazing paper [4] by relating the Hamiltonian of a Heisenberg's spin chain system with that of the dilatation operator in $N=4$ Supersymmetric Yang-Mills theory. So it seems that there is a close interplay between string theory, gauge theory and the spin-chain system, which makes the field of research more fascinating.

One of the interesting topic that has drawn lot of attention recently are the low lying spin chain system which correspond to the magnon excitation. Hofman and Maldacena have proposed [5] not too long back the correspondence of these magnon states with that of a specific configuration of semiclassical string states on $R \times S^{2}$ [3]. In particular the giant magnon solution of correspond to operators where one of the $S O(6)$ charge, $J$, is

\footnotetext{
${ }^{1}$ For some works considering integrability of sigma model on $A d S_{5} \times S^{5}$, see 21, 22, 23, 24, 25, 26, 27 , $28,29,30,31,18,32,33,34,35,36,37,38$. For reviews of this problems from different point of views, see [39, 40, 41, 42, 43, 44, 45$]$.
} 
taken to infinity, keeping the $E-J$ fixed $^{2}$. These excitations satisfy a dispersion relation of the type (in the large ' t Hooft limit $(\lambda)$ )

$$
E-J=\frac{\sqrt{\lambda}}{\pi}\left|\sin \frac{p}{2}\right|
$$

where $p$ is the magnon momentum. Hence after lot of work has been devoted to study and generalize to various other magnon states with two and three non vanishing momentum and so on, see for example [6, 7, 8, 9, 10, 11, 12, 13]. The basic idea in all these developments was to show what does this correspond to in the gauge theory side of the duality conjecture.

In the present paper we generalize the discussion of giant magnon in the NS5-brane background. In string theory NS5-brane solution is interesting because in the near horizon limit the theory on the worldvolume correspond to a nonlocal field theory, namely the little string theory (LST) ${ }^{3}$. Though the LST has been a misnomer since long time, it seems a good exercise to analyze the solution in various limits and find out the corresponding field theory. The near horizon NS5-brane world sheet theory is exactly solvable [14, so from the bulk view point it seems that the theory is integrable. A little is known about the boundary theory, hence from that prospective it is rather hard to make definite statements about the exact nature of the theory.

Motivated by the recent surge of interest in finding out the magnon solutions in anti-de sitter space, we study the NS5-brane worldvolume theory in near horizon limit. The near horizon limit, in a particular scaling yields a background with a linear dilaton. In order to study the magnon solution, one has to take proper care of the dilaton. We include the dilaton in the Polyakov action, which modifies obviously the worldsheet stress tensor. We show that even in the presence of a linear dilaton we get a giant magnon solution, and the exact dispersion relation for the magnon discussed in the literature.

Rest of the paper is organized as follows. In the section-2, we write down the Polyakov string in the near horizon limit of the NS 5-brane. We find out the magnon dispersion relation in the large $J$ sector. We get the dispersion relation in terms of total energy $E$, momentum along an angular direction $\left(\phi_{2}\right)$ of the sphere in the transverse direction of NS 5-brane $\left(P_{\phi_{2}}\right)$, momentum along the longitudinal directions of NS5-branes, $y^{i}$, and another conserved charge $D$. The conserved charge $D$ is related to the radial motion in the decoupling limit of NS5-brane background that, with appropriate choice of coordinates, is free and reflects the fact that the configuration of NS5-brane and fundamental string is BPS state.

In section-3, we try to study this situation further. We develop a Hamiltonian formalism for string in the near horizon region of $N$ NS5-branes with a general world-sheet metric. However due to the presence of the nontrivial coupling of the string to the dilaton, we take $\sqrt{\lambda} \rightarrow \infty$ to restrict to the study of the classical dynamics of string. Then in the first approximation we can neglect the coupling of the string to the dilaton in uniform gauge, and find the same magnon dispersion relation of section-2. Finally in section-4, we present our conclusion and outlook.

\footnotetext{
${ }^{2}$ The Hofman-Maldacena limit: $J \rightarrow \infty, \lambda=$ fixed, $p=$ fixed, $E-J=$ fixed

${ }^{3}$ For review, see 46, 47.
} 


\section{Polyakov string in the near horizon limit of NS5-brane background}

In this section we study the Polyakov string in the near horizon limit of the NS 5-brane background. The classical solution of N NS5-brane is given by

$$
\begin{aligned}
d s^{2} & =-d \tilde{t}^{2}+d y_{5}^{2}+H(r)\left(d r^{2}+r^{2} d \Omega_{3}^{2}\right) \\
e^{2\left(\Phi-\Phi_{0}\right)} & =H(r), \quad b=2 N \sin ^{2} \theta \cos \phi_{1} d \theta \wedge d \phi_{2}, \quad H(r)=1+\frac{N l_{s}^{2}}{r^{2}}
\end{aligned}
$$

where $y^{i}, i=6, \ldots, 9$ label world-volume directions of $N S 5$-branes, $H(r)$ is the harmonic function in the transverse space of the NS5-brane. Finally, $l_{s}$ is string length that is related to Regge slope as $l_{s}^{2}=\frac{1}{\alpha^{\prime}}$.

In the near horizon limit, $r \rightarrow 0$, one can ignore the 1 in $H(r)$, and solution would look like (defining $\left.\tilde{t}=\sqrt{N} l_{s} t\right)$

$$
d s^{2}=N l_{s}^{2}\left(-d t^{2}+d \theta^{2}+\sin ^{2} \theta d \phi_{1}^{2}+\sin ^{2} \theta \sin ^{2} \phi_{1} d \phi_{2}^{2}+\frac{d r^{2}}{r^{2}}\right)+d y_{5}^{2},
$$

where the metric on $S^{3}$ is written as

$$
d \Omega_{3}^{2}=d \theta^{2}+\sin ^{2} \theta d \phi_{1}^{2}+\sin ^{2} \theta \sin ^{2} \phi_{1} d \phi_{2}^{2} .
$$

At the same time

$$
e^{2 \Phi}=\frac{N l_{s}}{r^{2}}, \quad b_{\theta \phi_{2}}=2 N \sin ^{2} \theta \cos \phi_{1} .
$$

We are interested in the geometry (2.2), for finding out the giant magnon solution that is analogue of the giant magnon solution in $A d S_{5} \times S^{5}$ found in [5]. Our starting point is the Polyakov form of the string action in the background (2.2)

$$
\begin{aligned}
S & =-\frac{\sqrt{\lambda}}{4 \pi} \int_{-\pi}^{\pi} d \sigma d \tau\left[\sqrt{-\gamma} \gamma^{\alpha \beta} g_{M N} \partial_{\alpha} x^{M} \partial_{\beta} x^{N}-e^{\alpha \beta} \partial_{\alpha} x^{M} \partial_{\beta} x^{N} b_{M N}\right]+ \\
& +\frac{1}{4 \pi} \int_{-\pi}^{\pi} d \sigma d \tau \sqrt{-\gamma} R \Phi
\end{aligned}
$$

where the pre-factor $\sqrt{\lambda}$ is equal to

$$
\sqrt{\lambda}=N
$$

and where $\gamma^{\alpha \beta}$ is world-sheet metric and $R$ is its Ricci scalar. Further, $e^{\alpha \beta}$ is defined as $e^{01}=-e^{10}=1$. Finally, the modes $x^{M}, M=0, \ldots, 9$ parameterize the embedding of the string in the background (2.2). The variation of the action (2.5) with respect to $x^{M}$ implies following equations of motion

$$
\begin{array}{r}
-\frac{\sqrt{\lambda}}{4 \pi} \sqrt{-\gamma} \gamma^{\alpha \beta} \partial_{K} g_{M N} \partial_{\alpha} x^{M} \partial_{\beta} x^{N}+\frac{\sqrt{\lambda}}{2 \pi} \partial_{\alpha}\left[\sqrt{-\gamma} \gamma^{\alpha \beta} g_{K M} \partial_{\beta} x^{M}\right]- \\
-\frac{\sqrt{\lambda}}{2 \pi} \partial_{\alpha}\left[\epsilon^{\alpha \beta} \partial_{\beta} x^{M} b_{K M}\right]+\frac{\sqrt{\lambda}}{4 \pi} \epsilon^{\alpha \beta} \partial_{\alpha} x^{M} \partial_{\beta} x^{N} \partial_{K} b_{M N}+\frac{1}{4 \pi} \partial_{K} \Phi \sqrt{-\gamma} R=0 .
\end{array}
$$


Further, the variation of the action with respect to the metric implies the constraints 15]

$$
\begin{aligned}
-\frac{4 \pi}{\sqrt{-\gamma} \frac{\delta S}{\delta \gamma^{\alpha \beta}}} & =\sqrt{\lambda} g_{M N} \partial_{\alpha} x^{M} \partial_{\beta} x^{N}-R_{\alpha \beta}+ \\
& +\left(\nabla_{\alpha} \nabla_{\beta} x^{M}\right) \partial_{M} \Phi+\left(\partial_{\alpha} x^{M} \partial_{\beta} x^{N}\right) \partial_{M} \partial_{N} \Phi- \\
& -\frac{1}{2} \gamma_{\alpha \beta}\left(\sqrt{\lambda} \gamma^{\gamma \delta} \partial_{\gamma} x^{M} \partial_{\delta} x^{N} g_{M N}-R \Phi+2 \nabla^{\alpha} \nabla_{\alpha} \Phi\right)
\end{aligned}
$$

To proceed further it is convenient to introduce the variable $\rho$ that is related to $r$ as

$$
\rho=\ln \left(\frac{r}{\sqrt{N l_{s}^{2}}}\right) .
$$

Then in the conformal gauge $\gamma_{\alpha \beta}=\eta_{\alpha \beta}$ where $\eta_{\alpha \beta}=\operatorname{diag}(-1,1)$ and using the variable (2.9) the constraints (2.8) take simpler form

$$
\begin{aligned}
& T_{\sigma \sigma}=-4 \pi \frac{\delta S}{\delta \gamma^{\sigma \sigma}}=\frac{\sqrt{\lambda}}{2}\left(g_{M N} \partial_{\sigma} x^{M} \partial_{\sigma} x^{N}+g_{M N} \partial_{\tau} x^{M} \partial_{\tau} x^{N}\right)-\partial_{\tau}^{2} \rho \\
& T_{\tau \tau}=-4 \pi \frac{\delta S}{\delta \gamma^{\tau \tau}}=\frac{\sqrt{\lambda}}{2}\left(g_{M N} \partial_{\sigma} x^{M} \partial_{\sigma} x^{N}+g_{M N} \partial_{\tau} x^{M} \partial_{\tau} x^{N}\right)-\partial_{\sigma}^{2} \rho \\
& T_{\tau \sigma}=-4 \pi \frac{\delta S}{\delta \gamma^{\sigma \tau}}=\sqrt{\lambda} g_{M N} \partial_{\sigma} x^{M} \partial_{\tau} x^{N}-\partial_{\sigma} \partial_{\tau} \rho .
\end{aligned}
$$

Looking at the form of the background (2.2) and (2.4) we observe that the action (2.5) is invariant under following transformations of fields

$$
\begin{aligned}
t^{\prime}(\tau, \sigma) & =t(\sigma, \tau)+\epsilon_{t} \\
\phi_{2}^{\prime}(\tau, \sigma) & =\phi_{2}(\tau, \sigma)+\epsilon_{\phi_{2}}
\end{aligned}
$$

where $\epsilon_{t}, \epsilon_{\phi_{2}}$ are constants. Then it is a simple task to determine corresponding conserved charges

$$
\begin{aligned}
P_{t} & =\frac{\sqrt{\lambda}}{2 \pi} \int_{-\pi}^{\pi} d \sigma \sqrt{-\gamma} \gamma^{\tau \alpha} g_{t t} \partial_{\alpha} t \\
P_{\phi_{2}} & =\frac{\sqrt{\lambda}}{2 \pi} \int_{-\pi}^{\pi} d \sigma\left[\sqrt{-\gamma} \gamma^{\tau \alpha} g_{\phi_{2} \phi_{2}} \partial_{\alpha} \phi_{2}-\partial_{\alpha} \theta b_{\theta \phi_{2}}\right] .
\end{aligned}
$$

Note that $P_{t}$ is related to the energy as $P_{t}=-E$.

Moreover, the string action in the conformal gauge in the near horizon limit of NS5brane background is also invariant under following transformation

$$
r^{\prime}(\tau, \sigma)=\Lambda r(\tau, \sigma)
$$


for constant $\Lambda$. Alternatively, using the variable $\rho$ this symmetry is an ordinary translation symmetry

$$
\rho^{\prime}(\tau, \sigma)=\rho(\tau, \sigma)+\epsilon_{\rho}
$$

for constant $\epsilon_{\rho}$. This invariance leads to the existence of the third conserved charge

$$
D=\frac{\sqrt{\lambda}}{2 \pi} \int_{-\pi}^{\pi} d \sigma \sqrt{-\eta} \eta^{\tau \alpha} \frac{1}{r} \partial_{\alpha} r
$$

or

$$
D=\frac{\sqrt{\lambda}}{2 \pi} \int_{-\pi}^{\pi} d \sigma \sqrt{-\eta} \eta^{\tau \alpha} \partial_{\alpha} \rho
$$

Finally, the invariance of the metric with respect of the translation in $y^{i}$-directions implies an existence of five conserved charges

$$
P_{y_{i}}=\frac{\sqrt{\lambda}}{2 \pi} \int_{-\pi}^{\pi} d \sigma \sqrt{-\eta} \eta^{\tau \alpha} \partial_{\alpha} y^{i}
$$

Now we proceed to find the solution of the equations of motion given above which could be interpreted as an giant magnon. We closely follow very nice analysis presented in [16]. (See also [17]).

Let us now consider following ansatz for obtaining the giant magnon solution

$$
\begin{gathered}
t=-\frac{E}{\sqrt{\lambda}} \tau, \quad \theta=\theta(\sigma, \tau), \quad \rho=\rho(\tau), \\
\phi_{2}=\phi_{2}(\sigma, \tau), \quad \phi_{1}=\text { const. }, \quad y^{i}=v^{i} \tau .
\end{gathered}
$$

In conformal gauge the equation of motion for $\rho$ takes the form

$$
\partial_{\alpha}\left[\sqrt{-\eta} \eta^{\alpha \beta} \partial_{\beta} \rho\right]=0
$$

with the general solution

$$
\rho=C \tau+\rho_{0},
$$

where $C, \rho_{0}$ are constants. In order to assure that we are in the weak coupling region we have to demand that $C>0$ and also consider large $\rho_{0}$.

On the other hand the equation of motion for $t$ takes the form

$$
\partial_{\alpha}\left[\sqrt{-\eta} \eta^{\alpha \beta} g_{t t} \partial_{\beta} t\right]=0
$$

that is clearly solved with the ansatz 2.18). It can be also easily shown that the equations of motion for $y^{i}$ are solved with the ansatz (2.18). Further it is easy to see that the equation of motion for $\phi_{1}$ has two constant solutions:

$$
\phi_{1}=0, \quad \phi_{1}=\frac{\pi}{2} .
$$


Solution with $\phi_{1}=0$ corresponds to the trivial pint-like solution and we will not consider it further. We will restrict ourselves to the case when $\phi_{1}=\frac{\pi}{2}$. Note also that for $\phi_{1}=\frac{\pi}{2}$ the $b$ field vanishes.

Instead to solve the equations of motions for $x$ directly it is more convenient to solve the constraints (2.10) that can be interpreted as the first integrals of the theory. Inserting the ansatz (2.18) to the constraints coming from the variation of the metric (2.10) we obtain two equations

$$
\begin{aligned}
& -\frac{E^{2}}{\lambda}+C^{2}+v_{i} v^{i}+\sin ^{2} \theta\left(\dot{\phi}_{2}\right)^{2}+\dot{\theta}^{2}+\theta^{\prime 2}+\sin ^{2} \theta \phi_{2}^{\prime 2}=0, \\
& \dot{\phi}_{2} \phi_{2}^{\prime} \sin ^{2} \theta+\theta^{\prime} \dot{\theta}=0,
\end{aligned}
$$

where we have used (2.20) and also the notation $\partial_{\sigma} x=x^{\prime}, \partial_{\tau} x=\dot{x}$. Following [5, 16] we search for a solution with the boundary conditions

$$
\theta(\pi, \tau)-\theta(-\pi, \tau)=0, \quad \triangle \phi_{2}=\phi_{2}(\pi, \tau)-\phi_{2}(-\pi, \tau)=p,
$$

where $p$ is the momentum of the 'single magnon' excitation. Since the field $\phi_{2}$ does not satisfy periodic boundary conditions this solution corresponds to the open string.

As the next step we introduce the light-cone coordinate $\varphi$ through the formula

$$
\phi_{2}=\varphi_{2}+\omega \tau
$$

and take the ansatz for $\varphi_{2}$ and $\theta$ in the form

$$
\theta=\theta(\sigma-v \omega \tau), \quad \varphi_{2}=\varphi_{2}(\sigma-v \omega \tau) .
$$

With this ansatz eqn. (2.23) take the forms

$$
\begin{aligned}
& \left(\omega-v \omega \varphi_{2}^{\prime}\right) \varphi_{2}^{\prime} \sin ^{2} \theta-v \omega \theta^{\prime 2}=0, \\
& -\frac{E^{2}}{\lambda}+C^{2}+v_{i} v^{i}+\sin ^{2} \theta\left(\omega-v \omega \varphi_{2}^{\prime}\right)^{2}+\left(1+v^{2} \omega^{2}\right) \theta^{\prime 2}+\sin ^{2} \theta \varphi_{2}^{\prime 2}=0,
\end{aligned}
$$

where now $\theta^{\prime}, \varphi_{2}^{\prime}$ means derivative with respect of arguments of $\theta, \varphi_{2}$ respectively. If we now combine these equations we obtain

$$
\begin{aligned}
\varphi_{2}^{\prime} & =\frac{v\left(\frac{E^{2}}{\lambda}-C^{2}-v_{i} v^{i}-\omega^{2} \sin ^{2} \theta\right)}{\left(1-v^{2} \omega^{2}\right) \sin ^{2} \theta} \\
\theta^{\prime 2} & =\omega^{2} \frac{\left(\sin ^{2} \theta_{\max }-\sin ^{2} \theta\right)\left(\sin ^{2} \theta-\sin ^{2} \theta_{\min }\right)}{\left(1-v^{2} \omega^{2}\right)^{2} \sin ^{2} \theta},
\end{aligned}
$$

where

$$
\sin ^{2} \theta_{\max }=\frac{\frac{E^{2}}{\lambda}-C^{2}-v_{i} v^{i}}{\omega^{2}}, \quad \sin ^{2} \theta_{\min }=\left(\frac{E^{2}}{\lambda}-C^{2}-v_{i} v^{i}\right) v^{2}
$$


We see, in agreement with [16], that for this solution the derivative $\theta^{\prime}$ is finite everywhere and vanishes both at $\sin ^{2} \theta_{\max }$ and $\sin ^{2} \theta_{\text {min }}$. On the other hand using the arguments given there we can ague that the target space shape of the solution is determined by $\frac{d \theta}{d \varphi_{2}}$ that vanishes at $\theta=\theta_{\max }$ and diverges at $\theta=\theta_{\min }$.

We can also express the velocity $v$ in terms of $p$ by using the boundary condition for $\phi_{2}$ since

$$
\triangle \phi_{2}=\int_{-\pi}^{\pi} d \sigma \partial_{\sigma} \phi=\int_{-\pi}^{\pi} d \sigma \varphi_{2}^{\prime}=\int_{\theta_{\min }}^{\theta_{\max }} d \theta \frac{\varphi_{2}^{\prime}}{\left|\theta^{\prime}\right|}
$$

As the next step we insert (2.28) into the conserved charges given in (2.12) and we obtain

$$
\begin{aligned}
P_{t} & =\frac{\sqrt{\lambda}}{2 \pi} \int_{-\pi}^{\pi} d \sigma\left[\sqrt{-\eta} \eta^{\tau \alpha} g_{t t} \partial_{\alpha} t\right]=-E \\
P_{\phi_{2}} & =\frac{\sqrt{\lambda}}{2 \pi} \int_{-\pi}^{\pi} d \sigma\left[\sqrt{-\eta} \eta^{\tau \alpha} g_{\phi_{2} \phi_{2}} \partial_{\alpha} \phi_{2}-\partial_{\sigma} \theta b_{\theta \phi_{2}}\right]= \\
& =\frac{\sqrt{\lambda}}{\pi} \int_{z_{\min }}^{z_{\max }} d z \frac{\omega\left(1-z^{2}\right)}{\left|z^{\prime}\right|}\left(v \varphi_{2}^{\prime}-1\right),
\end{aligned}
$$

where we introduced the coordinate $z$ defined as

$$
1-z^{2}=\sin ^{2} \theta
$$

Finally we insert (2.18) into (2.15) and 2.17) and we obtain

$$
\begin{aligned}
& D=\frac{\sqrt{\lambda}}{2 \pi} \int_{-\pi}^{\pi} d \sigma \sqrt{-\eta} \eta^{\tau \alpha} \frac{1}{r} \partial_{\alpha} r=\sqrt{\lambda} C, \\
& P_{y_{i}}=\frac{\sqrt{\lambda}}{2 \pi} \int_{-\pi}^{\pi} d \sigma \sqrt{-\eta} \eta^{\tau \alpha} \partial_{\alpha} y^{i}=\sqrt{\lambda} v^{i} .
\end{aligned}
$$

The next goal is to explicitly integrate these expressions. Following the analysis given in [16] we easily get

$$
\begin{aligned}
p & =\Delta \phi_{2}=2 \int_{\theta_{\min }}^{\theta_{\max }} d \theta \frac{\varphi_{2}^{\prime}}{\left|\theta^{\prime}\right|}=2 \int_{z_{\min }}^{z_{\max }} \frac{d z}{\left|z^{\prime}\right|} \varphi_{2}^{\prime}= \\
& =2 v \omega \int_{z_{\min }}^{z_{\max }} d z \frac{z^{2}-z_{\min }^{2}}{\left(1-z^{2}\right) \sqrt{z_{\max }^{2}-z^{2}} \sqrt{z^{2}-z_{\min }^{2}}}= \\
& =-2 \frac{v \omega}{\sqrt{1-\left(\frac{E^{2}}{\lambda}-C^{2}-v_{i} v^{i}\right) v^{2}}} K(\eta)+\frac{2 \Pi\left(\frac{v^{2}\left(\frac{E^{2}}{\lambda}-C^{2}-v_{i} v^{i}\right)-1}{\left(\frac{E^{2}}{\lambda}-C^{2}-v_{i} v^{i}\right) v^{2}} \eta, \eta\right)}{\omega v \sqrt{1-\left(\frac{E^{2}}{\lambda}-C^{2}-v_{i} v^{i}\right) v^{2}}}
\end{aligned}
$$


where

$$
\begin{aligned}
z_{\text {max }}^{2} & =1-\sin ^{2} \theta_{\max }=1-\left(\frac{E^{2}}{\lambda}-C^{2}-v_{i} v^{i}\right) v^{2} \\
z_{\min }^{2} & =1-\sin ^{2} \theta_{\min }=1-\frac{\frac{E^{2}}{\lambda}-C^{2}-v_{i} v^{i}}{\omega^{2}} \\
\eta & =1-\frac{z_{\min }^{2}}{z_{\max }^{2}} .
\end{aligned}
$$

In the same way we can integrate $P_{\phi_{2}}$ with the result

$$
\begin{aligned}
P_{\phi_{2}} & =J=-2 \frac{\sqrt{\lambda}}{2 \pi} \int_{z_{\min }}^{z_{\max }} d z \frac{\omega\left(1-z^{2}\right)}{\left|z^{\prime}\right|}\left(1-v \varphi_{2}^{\prime}\right)= \\
& =-\frac{\omega}{|\omega|} \frac{\sqrt{\lambda}}{\pi} \sqrt{1-v^{2}\left(\frac{E^{2}}{\lambda}-C^{2}-v_{i} v^{i}\right)}(K(\eta)-E(\eta)) .
\end{aligned}
$$

Note that the functions $E(\eta), K(\eta)$, and $\Pi$ are defined by formulas (We follow the notation given in [16].)

$$
\begin{aligned}
& I_{1}=\int_{z_{\min }}^{z_{\max }} d z \frac{1}{\sqrt{z^{2}-z_{\min }^{2}} \sqrt{z_{\max }^{2}-z^{2}}}=\frac{1}{z_{\max }} K(\eta), \\
& I_{2}=\int_{z_{\min }}^{z_{\max }} d z \frac{z^{2}}{\sqrt{z^{2}-z_{\min }^{2}} \sqrt{z_{\max }^{2}-z^{2}}}=z_{\max } E(\eta), \\
& I_{3}=\int_{z_{\min }}^{z_{\max }} d z \frac{1}{\left(1-z^{2}\right) \sqrt{z^{2}-z_{\min }^{2}} \sqrt{z_{\max }^{2}-z^{2}}}=\frac{1}{z_{\max }\left(1-z_{\max }^{2}\right)} \Pi\left(\frac{z_{\max }^{2}-z_{\min }^{2}}{z_{\max }^{2}-1}, \eta\right) .
\end{aligned}
$$

The relation (2.36) allows us to express the modulus $\eta$ in terms of $J \equiv P_{\phi_{2}}, E, v, C$ and $v_{i}$. On the other hand it is clear that there is no analytic expression for the dispersion relation. However we would like to analyze these equations for large values of charge $J$.

\subsection{Infinite $J$ Giant Magnon}

To find more illuminating result we restrict to the case of an infinite $J$ giant magnon. This situation occurs for $z_{\min } \rightarrow 0$ that corresponds to

$$
\omega^{2}=\frac{E^{2}}{\lambda}-C^{2}-v_{i} v^{i}
$$

In fact, it is easy to see that for $z_{\min } \rightarrow 0$ the charge (2.36) really diverges. We also choose $\omega=-\sqrt{\frac{E^{2}}{\lambda}-C^{2}-v_{i} v^{i}}$ in order to have positive, large $J$. 
Our goal now is to find the dispersion relation between $E, J$ and possibly another charges that is an analogue of the relation found in [5]. It turns out that the relevant dispersion relation takes the form

$$
\begin{aligned}
& \sqrt{E^{2}-D^{2}-\left(P_{y_{i}}\right)^{2}}-J=\frac{\sqrt{\lambda}}{\pi} \int_{0}^{z_{\max }} d z \frac{z}{\sqrt{z_{\max }^{2}-z^{2}}}= \\
= & \frac{\sqrt{\lambda}}{\pi} \sqrt{1-\left(\frac{E^{2}}{\lambda}-C^{2}-v_{i} v^{i}\right) v^{2}} .
\end{aligned}
$$

As the next step we calculate the momentum defined (2.34) for $z_{\min }=0$

$$
p=2 v \omega \int_{0}^{z_{\max }} d z \frac{z}{\left(1-z^{2}\right) \sqrt{z_{\max }^{2}-z^{2}}}=2 \cos ^{-1} v \sqrt{\frac{E^{2}}{\lambda}-C^{2}-v_{i} v^{i}} .
$$

Then finally using (2.40) we can rewrite the dispersion relation (2.39) into a more natural form

$$
\sqrt{E^{2}-D^{2}-\left(P_{y_{i}}\right)^{2}}-J=\frac{\sqrt{\lambda}}{\pi}\left|\sin \frac{p}{2}\right|
$$

A comment is in order for the magnon dispersion relation (2.41). We see that there is a natural symmetry between $D$ and $P_{y_{i}}$. It is clear that we could generalize the dispersion relation for giant magnon in $A d S_{5} \times S^{5}$ in the same way when we include the motion of the string along the $A d S_{5}$ boundary. In other words the presence of the charge $D$ does not imply any new physical interpretation of the dispersion relation given above. It simply reflects the fact that the motion of the string along the radial direction in the near horizon limit of $N S 5$-brane background is free.

\section{Uniform light-cone gauge}

In this section, we would like to investigate the giant magnon-like dispersion relation (2.41) for the open string orbiting in the background of N NS 5-brane in more details. For example, does the giant magnon solution pose uniform distribution of the angular momentum along the string world-sheet as in case of giant magnon in $A d S_{5} \times S^{5}$ [16] ? To answer this question we have to develop the Hamiltonian formalism for string in the near horizon region of $N$ NS5-branes with general world-sheet metric. However this requirement makes the analysis very obscure due to the presence of the non-trivial coupling of the string to the dilaton. On the other hand when we restrict to the study of the classical dynamics of string we have to take $\sqrt{\lambda} \rightarrow \infty$. Then in the first approximation we can neglect the coupling of the string to the dilaton when we will try to find the solution in uniform gauge. Let us proceed along this line.

We again start with the action

$$
S=-\frac{\sqrt{\lambda}}{4 \pi} \int_{-\pi}^{\pi} d \sigma d \tau\left[\sqrt{-\gamma} \gamma^{\alpha \beta} g_{M N} \partial_{\alpha} x^{M} \partial_{\beta} x^{N}-\epsilon^{\alpha \beta} \partial_{\alpha} x^{M} \partial_{\beta} x^{N} b_{M N}\right] .
$$


To implement the uniform light-cone we will follow [18]. Using (3.1) we determine the momenta $p_{M}$ conjugate to $x^{M}$

$$
p_{M}=-\frac{\sqrt{\lambda}}{2 \pi}\left(\sqrt{-\gamma} \gamma^{\tau \alpha} g_{M N} \partial_{\beta} x^{N}-b_{M N} \partial_{\sigma} x^{N}\right)
$$

Let us also use following parametrization of the metric variables $\gamma_{\mu \nu}$

$$
\lambda^{ \pm}=\frac{\sqrt{-\gamma} \pm \gamma_{\tau \sigma}}{\gamma_{\sigma \sigma}}, \quad \xi=\ln \gamma_{\sigma \sigma}
$$

where $\lambda^{ \pm}$are manifestly invariant under the Weyl transformations of metric

$$
\gamma_{\alpha \beta}^{\prime}(\sigma, \tau)=e^{\phi(\tau, \sigma)} \gamma_{\alpha \beta}(\tau, \sigma)
$$

while $\xi$ transforms as $\xi^{\prime}(\sigma, \tau)=\xi(\sigma, \tau)+\phi(\sigma, \tau)$. Then since the action does not contain the time-derivative of the metric we find that the momenta conjugate to $\lambda^{ \pm}, \xi$ are zero:

$$
\pi_{ \pm}=\frac{\delta S}{\delta \partial_{\tau} \lambda^{ \pm}}=0, \quad \pi_{\xi}=\frac{\delta S}{\delta \partial_{\tau} \xi}=0
$$

and form the primary constraints of the theory. As the next step we determine the Hamiltonian density

$$
\begin{aligned}
\mathcal{H}_{0} & =\pi_{+} \partial_{\tau} \lambda^{+}+\pi_{-} \partial_{\tau} \lambda^{-}+\pi_{\xi} \partial_{\tau} \xi+\partial_{\tau} x^{M} p_{M}-\mathcal{L}= \\
& =-\frac{1}{\sqrt{-\gamma} \gamma^{\tau \tau}}\left[\frac{\pi}{\sqrt{\lambda}} p_{M} g^{M N} p_{N}+\frac{\sqrt{\lambda}}{4 \pi} g_{M N} \partial_{\sigma} x^{M} \partial_{\sigma} x^{N}+p_{M} g^{M N} b_{N K} \partial_{\sigma} x^{K}+\right. \\
& \left.+\frac{\sqrt{\lambda}}{4 \pi} \partial_{\sigma} x^{M} b_{M N} g^{N K} b_{K L} \partial_{\sigma} x^{L}\right]-\frac{\gamma^{\tau \sigma}}{\gamma^{\tau \tau}} p_{M} \partial_{\sigma} x^{M}
\end{aligned}
$$

or alternatively using the variables (3.3)

$$
\begin{aligned}
& \mathcal{H}_{0}=\frac{\lambda^{+}+\lambda^{-}}{2} T_{0}+\frac{\lambda^{+}-\lambda^{-}}{2} T_{1}=\lambda^{+} T_{+}+\lambda^{-} T_{-}, \\
& T_{+}=\frac{1}{2}\left(T_{0}+T_{1}\right), \quad T_{-}=\frac{1}{2}\left(T_{0}-T_{1}\right),
\end{aligned}
$$

where

$$
\begin{aligned}
T_{0} & =\left[\frac{\pi}{\sqrt{\lambda}} p_{M} g^{M N} p_{N}+\frac{\sqrt{\lambda}}{4 \pi} g_{M N} \partial_{\sigma} x^{M} \partial_{\sigma} x^{N}+p_{M} g^{M N} b_{N K} \partial_{\sigma} x^{K}+\right. \\
& \left.+\frac{\sqrt{\lambda}}{4 \pi} \partial_{\sigma} x^{M} b_{M N} g^{N K} b_{K L} \partial_{\sigma} x^{L}\right] \\
T_{1} & =\partial_{\sigma} x^{M} p_{M}
\end{aligned}
$$

The stability of the primary constraints imply the secondary ones 48, 49, 50]

$$
T_{0}=T_{1}=0
$$


It can be shown that stability of these constraints does not imply any additional ones.

Note also that the invariance of the action (3.1) under the shift $\phi_{2}^{\prime}=\phi_{2}+\epsilon$ implies an existence of the conserved quantity

$$
P_{\phi_{2}}=\frac{\sqrt{\lambda}}{2 \pi} \int_{-\pi}^{\pi} d \sigma\left[\sqrt{-\gamma} \gamma^{\tau \alpha} g_{\phi_{2} N} \partial_{\alpha} x^{N}-\epsilon^{\tau \alpha} b_{\phi_{2} N} \partial_{\alpha} x^{N}\right]=-\int_{-\pi}^{\pi} d \sigma p_{\phi_{2}} .
$$

Our goal is to develop the Hamiltonian formulation of completely fixed gauge theory. Note that the momenta $\pi_{ \pm}$and $\pi_{\xi}$ decouple from the theory and these constraints can be ignored. Then we can interpret $\lambda^{ \pm}$as the Lagrange multipliers for the constraints $T_{ \pm}$. We fix the gauge symmetry generated by $T_{0}, T_{1}$ if we impose following uniform light-cone gauge [18]

$$
p_{\phi_{2}}=\frac{1}{2 \pi} J, \quad t=\tau .
$$

The fixing the gauge implies that the original Hamiltonian $H_{0}=\int_{-\pi}^{\pi} d \sigma \mathcal{H}_{0}$ strongly vanishes. Secondly, the stability of the gauge fixing functions (3.11) determine the values of $\lambda_{ \pm}$. However the explicit values of these constraints are not important for our purposes.

The fact that $T_{0}, T_{1}$ strongly vanish allows us to find the dynamics on the reduced phase space. Firstly, from $T_{1}$ we express $\partial_{\sigma} \phi_{2}$ as

$$
\partial_{\sigma} \phi_{2}=-\frac{1}{p_{\phi_{2}}} \partial_{\sigma} x^{m} p_{m}
$$

where $m$ now label all coordinates different from $t, \phi_{2}$. Note that $x^{m}, p_{m}$ parameterize the reduced phase space.

Secondly, the gauge fixing (3.11) implies that the Hamiltonian that governs the dynamics on the reduced phase space should be identified with $-p_{t}$. This follows from the fact that for the gauge fixing (3.11) the action takes the form

$$
S=\int d \tau d \sigma\left[\dot{x}^{m} p_{m}+p_{t}\right]
$$

that suggests that the Hamiltonian density on reduced phase space $\mathcal{H}$ should be identified with $-p_{t}$. Then the time evolution of $p_{m}, x^{m}$ is governed by following equation

$$
\partial_{\tau} p_{m}=\left\{p_{m}, H\right\}_{D}, \quad \partial_{\tau} x^{m}=\left\{x^{m}, H\right\}_{D},
$$

where $H=\int_{-\pi}^{\pi} d \sigma \mathcal{H}$ and where subscript $D$ means Dirac bracket that however for the gauge fixing (3.11) coincides with the original Poisson brackets

$$
\left\{x^{m}(\sigma), p_{n}\left(\sigma^{\prime}\right)\right\}=\delta_{n}^{m} \delta\left(\sigma-\sigma^{\prime}\right) .
$$

As the next step we express $p_{t}$ as functions of the canonical variables $p_{m}, x^{m}$ with the help of $T_{1}$ and $T_{0}$

$$
\begin{aligned}
p_{t}^{2} & =\frac{\sqrt{\lambda}}{\pi}\left(\frac{\pi}{\sqrt{\lambda}} p_{m} g^{m n} p_{n}+\frac{\pi}{\sqrt{\lambda}} p_{\phi_{2}}^{2} g^{\phi_{2} \phi_{2}}+\frac{\sqrt{\lambda}}{4 \pi} \partial_{\sigma} x^{m} g_{m n} \partial_{\sigma} x^{n}+\frac{\sqrt{\lambda}}{4 \pi} \frac{g_{\phi_{2} \phi_{2}}}{p_{\phi_{2}}^{2}}\left(p_{m} \partial_{\sigma} x^{m}\right)^{2}-\right. \\
& -p_{\theta} g^{\theta \theta} b_{\theta \phi_{2}} \frac{1}{p_{\phi_{2}}} \partial_{\sigma} x^{m} p_{m}+p_{\phi_{2}} g^{\phi_{2} \phi_{2}} b_{\phi_{2} \theta} \partial_{\sigma} \theta+ \\
& \left.+\frac{\sqrt{\lambda}}{4 \pi} \partial_{\sigma} \theta b_{\theta \phi_{2}} g^{\phi_{2} \phi_{2}} b_{\phi_{2} \theta} \partial_{\sigma} \theta+\frac{\sqrt{\lambda}}{4 \pi p_{\phi_{2}}^{2}}\left(p_{m} \partial_{\sigma} x^{m}\right) b_{\phi_{2} \theta} g^{\theta \theta} b_{\theta \phi_{2}}\left(p_{m} \partial_{\sigma} x^{m}\right)\right) \equiv \mathbf{K} .
\end{aligned}
$$


Then the Hamiltonian density $\mathcal{H}$ takes the form

$$
\mathcal{H}=\sqrt{\mathbf{K}}
$$

where we have picked up the negative root in the equation $p_{t}^{2}=\mathbf{K}[18$.

To simplify the analysis further note that five coordinates $y^{i}, i=0, \ldots, 5$ and the corresponding momenta $p_{y_{i}}$ that determine the dynamics of the string along the worldvolume of NS5-branes are free. Then we can solve their equations of motion by requirement that $y^{i}$ are constant and $p_{y_{i}}=0$. Then the remaining degrees of freedom are

$$
\left(r, p_{r}\right), \quad\left(\theta, p_{\theta}\right), \quad\left(\phi_{1}, p_{\phi_{1}}\right)
$$

and we presume that they spatial and time dependence has following form

$$
\begin{aligned}
r & =r(\tau), \quad p_{r}=p_{r}(\tau), \\
\phi_{1} & =\phi_{1}^{\text {const }}, \quad p_{\phi_{1}}=0, \\
\theta & =\theta(\sigma-v \tau), \quad p_{\theta}=p_{\theta}(\sigma-v \tau) .
\end{aligned}
$$

First of all let us determine the values of $\phi_{1}$. The consistency of the ansatz (3.19) implies that $\phi_{1}=\frac{\pi}{2}$. However for this value the NS two form field (2.4) vanishes. In what follows we use the notation $g_{\phi_{2} \phi_{2}} \equiv g_{\phi_{2} \phi_{2}}\left(\phi_{1}=\pi / 2\right)=\sin ^{2} \theta$. Further, the equations of motion for $p_{r}, r$ take the form

$$
\partial_{\tau} r=\frac{p_{r} r^{2}}{\sqrt{\mathbf{K}}}, \quad \partial_{\tau} p_{r}=-\frac{p_{r}^{2} r}{\sqrt{\mathbf{K}}}
$$

that imply

$$
\partial_{\tau}\left(p_{r}^{2} r^{2}\right)=0 \text {. }
$$

In other words the expression $p_{r}^{2} g^{r r}$ that is presented in $\mathbf{K}$ is constant. Then the function $\mathbf{K}$ takes the form

$$
\mathbf{K}=\frac{\sqrt{\lambda}}{\pi}\left(\frac{\pi}{\sqrt{\lambda}} p_{r}^{2} g^{r r}+\frac{\pi}{\sqrt{\lambda}} p_{\phi_{2}}^{2} g^{\phi_{2} \phi_{2}}+\frac{\pi}{\sqrt{\lambda}} p_{\theta}^{2} g^{\theta \theta}+\frac{\sqrt{\lambda}}{4 \pi} \partial_{\sigma} \theta^{\prime 2} g_{\theta \theta}+\frac{\sqrt{\lambda}}{4 \pi} \frac{g_{\phi_{2} \phi_{2}}}{p_{\phi_{2}}^{2}}\left(p_{\theta} \partial_{\sigma} \theta\right)^{2}\right),
$$

where we have used the fact that $b_{\phi_{2} \theta}\left(\phi_{1}=\frac{\pi}{2}\right)=0$. Note also that the equation of motion for $\theta$ takes the form

$$
\partial_{\tau} \theta=\frac{1}{\sqrt{\mathbf{K}}}\left(p_{\theta} g^{\theta \theta}+\frac{\lambda}{4 \pi^{2}} \frac{g_{\phi_{2} \phi_{2}}}{p_{\phi_{2}}^{2}}\left(\partial_{\sigma} \theta p_{\theta} \partial_{\sigma} \theta\right)\right)
$$

Since the problem reduces to the study of the dynamics of $\theta$ and $r$ only it is more natural to proceed to the Lagrangian formalism. Using (3.20) and (3.23) and after some algebra we find the Lagrangian density defined as

$$
\mathcal{L}=\partial_{\tau} r p_{r}+\partial_{\tau} \theta p_{\theta}-\sqrt{\mathbf{K}}
$$


takes the form

$$
\mathcal{L}=-p_{\phi_{2}} \sqrt{\left[g^{\phi_{2} \phi_{2}}+\frac{\lambda}{4 \pi^{2}} \frac{1}{p_{\phi_{2}}^{2}}\left(\partial_{\sigma} \theta\right)^{2}\right]\left[1-\left(\partial_{\tau} r\right)^{2} g_{r r}\right]-g^{\phi_{2} \phi_{2}} g_{\theta \theta}^{2}\left(\partial_{\tau} \theta\right)^{2}} .
$$

As the next step we insert the ansatz $\theta=\theta(\sigma-v \tau)$ into (3.25). Since $\dot{R}=C^{\prime} R$ we obtain that (3.25) takes the form

$$
\mathcal{L}_{\text {red }}=-\sqrt{g^{\phi_{2} \phi_{2}} p_{\phi_{2}}^{2}\left(1-C^{\prime 2}\right)-\left(p_{\phi_{2}}^{2} v^{2} g^{\phi_{2} \phi_{2}}-\left(1-C^{\prime 2}\right) \frac{\lambda}{4 \pi^{2}}\right) \theta^{\prime 2}} .
$$

In other words the problem reduces to the dynamics of one degree of freedom $\theta$ that depends on the parameter $u \equiv \sigma-v \tau$. Then it is convenient to find the corresponding Hamiltonian since using the fact that (3.26) does not explicitly depend on $u$ the corresponding Hamiltonian, that determines the evolution with respect to the parameter $u$ is conserved. Explicitly, the conjugate momentum to $\theta$ takes the form

$$
\mathbf{p}=\frac{\delta \mathcal{L}}{\delta \theta^{\prime}}=\frac{\left(p_{\phi_{2}}^{2} v^{2} g^{\phi_{2} \phi_{2}}+\left(C^{\prime 2}-1\right) \frac{\lambda}{4 \pi^{2}}\right) \theta^{\prime}}{\sqrt{(\ldots)}}
$$

and hence the reduced Hamiltonian is equal to

$$
H^{r e d}=\theta^{\prime} \mathbf{p}-\mathcal{L}^{r e d}=\frac{g^{\phi_{2} \phi_{2}} p_{\phi_{2}}^{2}\left(1-C^{\prime 2}\right)}{\sqrt{(\ldots)}} .
$$

Since the reduced Hamiltonian obeys $\frac{d H^{\text {red }}}{d u}=0$ we set it to some constant

$$
H^{\text {red }}=R \text {. }
$$

Then from (3.28) we can find the differential equation for $\theta^{\prime}$

$$
\theta^{\prime 2}=\frac{4 \pi^{2} p_{\phi_{2}}^{2}}{\lambda \sin ^{2} \theta} \frac{\sin ^{2} \theta-\frac{p_{\phi_{2}}^{2}\left(1-C^{\prime 2}\right)}{R^{2}}}{\frac{4 \pi^{2} p_{\phi_{2}}^{2} v^{2}}{\lambda\left(1-C^{\prime 2}\right)}-\sin ^{2} \theta}
$$

The equation (3.30) implies following bound on the allowed values of $\theta$

$$
\frac{p_{\phi}^{2}\left(1-C^{\prime 2}\right)}{R}<\sin ^{2} \theta<\frac{4 \pi^{2}}{\lambda} \frac{p_{\phi_{2}}^{2} v^{2}}{1-C^{\prime 2}} .
$$

It is again convenient to introduce the variable $z^{2}=1-\sin ^{2} \theta$ that now obeys

$$
\begin{aligned}
z^{\prime 2} & =\frac{4 \pi^{2} p_{\phi_{2}}^{2}}{\lambda} \frac{z^{2}-z_{\min }^{2}}{z_{\max }^{2}-z^{2}}, \quad z_{\min } \leq z \leq z_{\max }, \\
z_{\min }^{2} & =1-\frac{p_{\phi_{2}}^{2}\left(1-C^{\prime 2}\right)}{R^{2}}, \quad z_{\max }^{2}=1-\frac{4 \pi^{2}}{\lambda} \frac{p_{\phi_{2}}^{2} v^{2}}{\left(1-C^{\prime 2}\right)} .
\end{aligned}
$$


We see from (3.32) that the case of infinite giant magnon corresponding to $z_{\min }=0$ occurs for $R^{2}=p_{\phi_{2}}^{2}\left(1-C^{2}\right)$.

Let us now calculate $p_{t}$ for this case. Since $p_{t}=-\sqrt{\mathbf{K}}$ we obtain

$$
p_{t}=-\frac{R}{\left(1-C^{\prime 2}\right)} \frac{z_{\max }^{2}-z_{\min }^{2}}{\sqrt{z_{\max }^{2}-z^{2}}} \frac{1}{\sqrt{\left(z_{\max }^{2}-z^{2}\right)}}
$$

and hence

$$
\frac{p_{t}}{\left|z^{\prime}\right|}=-\frac{\sqrt{\lambda}}{2 \pi} \frac{R}{\left(1-C^{\prime 2}\right) p_{\phi_{2}}} \frac{z_{\max }^{2}-z_{\min }^{2}}{\sqrt{\left(z_{\max }^{2}-z^{2}\right)\left(z^{2}-z_{\min }^{2}\right)}} .
$$

Now it turns out that the dispersion relation has following form

$$
E-\frac{J}{\sqrt{1-C^{\prime 2}}}=\frac{\sqrt{\lambda}}{\pi} \frac{1}{\sqrt{1-C^{\prime 2}}} \sqrt{1-\frac{4 \pi^{2} p_{\phi_{2}}^{2} v^{2}}{\lambda\left(1-C^{\prime 2}\right)}}=\frac{1}{\sqrt{1-C^{\prime 2}}} \frac{\sqrt{\lambda}}{\pi}\left|\sin \frac{p}{2}\right|
$$

using the fact that

$$
\begin{aligned}
& p=\triangle \phi_{2}=\int_{-\pi}^{\pi} d \sigma \partial_{\sigma} \phi_{2}=-\frac{1}{p_{\phi_{2}}} \int_{-\pi}^{\pi} d \sigma p_{\theta} \partial_{\sigma} \theta= \\
& =\frac{2 \pi v}{p_{\phi_{2}}} \frac{R}{\left(1-C^{2}\right)} \int_{z_{\min }}^{z_{\max }} \frac{d z}{1-z^{2}} \frac{2 \pi p_{\phi_{2}}}{\sqrt{\lambda}} \frac{\sqrt{z^{2}-z_{\min }^{2}}}{\sqrt{z_{\max }^{2}-z^{2}}}
\end{aligned}
$$

that for $z_{\min }=0$ implies

$$
\cos \frac{p}{2}=\frac{2 \pi}{\sqrt{\lambda}} \frac{p_{\phi_{2}} v}{\sqrt{1-C^{2}}} .
$$

Note that we have also presumed $-p_{\theta} \partial_{\sigma} \theta^{\prime}>0$ so that $\partial_{\sigma} \theta^{\prime}<0$. We have to find the relation between $C^{\prime}$ and the conserved dilaton charge defined in (2.16). To do this note that the equation of motion for $r$ is

$$
\partial_{\tau} r=\frac{g^{r r} p_{r}}{\sqrt{\mathbf{K}}}
$$

Note that we have defined $C^{\prime}$ through the relation $\partial_{\tau} r=C^{\prime} r$. Then (3.38) implies

$$
p_{r} r=C^{\prime} \sqrt{\mathbf{K}}=-C^{\prime} p_{t} .
$$

On the other hand the dilaton charge is equal to

$$
D=\int_{-\pi}^{\pi} d \sigma p_{r} r
$$

so that using (3.39) we finally obtain

$$
D=C^{\prime} E
$$


Then we can rewrite the relation (3.35) in more suggestive form

$$
\sqrt{E^{2}-D^{2}}-J=\frac{\sqrt{\lambda}}{\pi}\left|\sin \frac{p}{2}\right|
$$

that coincides with the result given in (2.41) for $P_{y_{i}}=0$.

\section{Conclusions}

In this paper we have studied the giant magnon solution in the near horizon limit of NS 5-branes. We derive the magnon like dispersion relation in Polyakov action of the string in the conformal gauge. For large $J$ value, we have been able to compute the one magnon dispersion relation in this background. We further have examined the magnon solution in the Hamiltonian formalism in the uniform gauge. The presence of the nontrivial dilaton makes the formalism obscure, but one can show that there in the limit of $\sqrt{\lambda} \rightarrow \infty$, the term in the action which determines the coupling of dilaton becomes very small, and one can ignore this. By making use of the Hamiltonian formalism we have been able to reduce it to the dynamics of one degree of freedom. We have been able to find out the magnon dispersion relation which matches exactly with the one we find in the Polyakov action. Moreover, we have argued that this dispersion relation takes the same form as the dispersion relation in case of giant magnon in $A d S_{5} \times S^{5}$ when we consider the motion along the boundary of $A d S_{5}$. This follows from the fact that the motion of the fundamental string in the near horizon region of NS5-branes is free and it is a reflection of fact that the configuration of the NS5-brane and fundamental string is a BPS state.

The present analysis can be extended in various directions. First of all it would be very interesting and challenging to find the boundary theory analogue of the present study. It is not clear whether the theory is integrable from the boundary theory. But given the result of the present paper it is interesting to find out operators in the LST that correspond to the giant magnons in the NS5-brane near horizon geometry. Further one can try to study the connection of this particular giant magnon solution to the pp-wave of the NS5-brane background, which is the Nappi-Witten model in the lines of 19.

Acknowledgements: We would like to thank S. Minwalla, J. Govaerts and W. H. Huang for useful correspondence. RRN would like to thank Bum-Hoon Lee and Sunggeun Lee for interesting discussions. The work of JK was supported in part by the Czech Ministry of Education under Contract No. MSM 0021622409, by INFN, by the MIUR-COFIN contract 2003-023852, by the EU contracts MRTN-CT-2004-503369 and MRTN-CT-2004-512194, by the INTAS contract 03-516346 and by the NATO grant PST.CLG.978785. The work of RRN was supported by the Science Research Center Program of the Korean Science and Engineering Foundation through the Center for Quantum Spacetime (CQUeST) of Sogang University grant no. R11-2005-021. 


\section{References}

[1] J. M. Maldacena, "The large N limit of superconformal field theories and supergravity," Adv. Theor. Math. Phys. 2, 231 (1998) [Int. J. Theor. Phys. 38, 1113 (1999)] [arXiv:hep-th/9711200].

[2] D. Berenstein, J. M. Maldacena and H. Nastase, "Strings in flat space and pp waves from $N$ = 4 super Yang Mills," JHEP 0204, 013 (2002) [arXiv:hep-th/0202021].

[3] S. S. Gubser, I. R. Klebanov and A. M. Polyakov, "A semi-classical limit of the gauge/string correspondence," Nucl. Phys. B 636, 99 (2002) [arXiv:hep-th/0204051].

[4] J. A. Minahan and K. Zarembo, "The Bethe-ansatz for $N=4$ super Yang-Mills," JHEP 0303, 013 (2003) [arXiv:hep-th/0212208].

[5] D. M. Hofman and J. M. Maldacena, “Giant magnons," J. Phys. A 39, 13095 (2006) [arXiv:hep-th/0604135].

[6] N. Dorey, "Magnon bound states and the AdS/CFT correspondence," J. Phys. A 39, 13119 (2006) [arXiv:hep-th/0604175].

[7] J. A. Minahan, A. Tirziu and A. A. Tseytlin, "Infinite spin limit of semiclassical string states," JHEP 0608, 049 (2006) [arXiv:hep-th/0606145].

[8] H. Y. Chen, N. Dorey and K. Okamura, "Dyonic giant magnons,” JHEP 0609, 024 (2006) [arXiv:hep-th/0605155].

[9] C. S. Chu, G. Georgiou and V. V. Khoze, "Magnons, classical strings and beta-deformations," JHEP 0611, 093 (2006) [arXiv:hep-th/0606220].

[10] M. Spradlin and A. Volovich, "Dressing the giant magnon," JHEP 0610, 012 (2006) [arXiv:hep-th/0607009].

[11] N. P. Bobev and R. C. Rashkov, "Multispin giant magnons," Phys. Rev. D 74, 046011 (2006) [arXiv:hep-th/0607018].

[12] S. Ryang, “Three-spin giant magnons in AdS(5) x $S^{* * 5}$," JHEP 0612, 043 (2006) [arXiv:hep-th/0610037].

[13] W. H. Huang, "Giant magnons under NS-NS and Melvin fields,” JHEP 0612, 040 (2006) [arXiv:hep-th/0607161].

[14] C. G. . Callan, S. B. Giddings, J. A. Harvey and A. Strominger, "Evanescent black holes," Phys. Rev. D 45 (1992) 1005 [arXiv:hep-th/9111056].

[15] R. E. Rudd, "Light cone gauge quantization of 2-D sigma models," Nucl. Phys. B 427 (1994) 81 [arXiv:hep-th/9402106].

[16] G. Arutyunov, S. Frolov and M. Zamaklar, "Finite-size effects from giant magnons," arXiv:hep-th/0606126.

[17] D. Astolfi, V. Forini, G. Grignani and G. W. Semenoff, "Gauge invariant finite size spectrum of the giant magnon," arXiv:hep-th/0702043.

[18] G. Arutyunov and S. Frolov, "Integrable Hamiltonian for classical strings on AdS(5) X S(5)," JHEP 0502 (2005) 059 [arXiv:hep-th/0411089].

[19] J. Maldacena and I. Swanson, "Connecting giant magnons to the pp-wave: An interpolating limit of $A d S(5) X S(5), "$ arXiv:hep-th/0612079. 
[20] I. Bena, J. Polchinski and R. Roiban, "Hidden symmetries of the AdS(5) x $S^{* * 5}$ superstring," Phys. Rev. D 69 (2004) 046002 [arXiv:hep-th/0305116].

[21] J. Kluson, "BRST invariance of non-local charges and monodromy matrix of bosonic string on $\operatorname{AdS}(5) \times S^{* *} 5, "$ arXiv:hep-th/0701013.

[22] N. Dorey and B. Vicedo, "A symplectic structure for string theory on integrable backgrounds," arXiv:hep-th/0606287.

[23] N. Gromov, V. Kazakov, K. Sakai and P. Vieira, "Strings as multi-particle states of quantum sigma-models," Nucl. Phys. B 764 (2007) 15 [arXiv:hep-th/0603043].

[24] L. F. Alday, G. Arutyunov and S. Frolov, "Green-Schwarz strings in TsT-transformed backgrounds," JHEP 0606 (2006) 018 [arXiv:hep-th/0512253].

[25] A. Das, A. Melikyan and M. Sato, "The algebra of flat currents for the string on AdS(5) $x$ $S^{* *} 5$ in the light-cone gauge," JHEP 0511 (2005) 015 [arXiv:hep-th/0508183].

[26] L. F. Alday, G. Arutyunov and S. Frolov, "New integrable system of 2dim fermions from strings on $A d S(5) x S^{* *}$," JHEP 0601 (2006) 078 [arXiv:hep-th/0508140].

[27] G. Arutyunov and M. Zamaklar, "Linking Baecklund and monodromy charges for strings on $A d S(5) x S^{* *} 5$," JHEP 0507 (2005) 026 [arXiv:hep-th/0504144].

[28] S. Frolov, "Lax pair for strings in Lunin-Maldacena background," JHEP 0505 (2005) 069 [arXiv:hep-th/0503201].

[29] B. Chen, Y. L. He, P. Zhang and X. C. Song, "Flat currents of the Green-Schwarz superstrings in $A d S(5) x S^{* *} 1$ and $A d S(3) x S^{* *} 3$ backgrounds," Phys. Rev. D 71 (2005) 086007 [arXiv:hep-th/0503089].

[30] L. F. Alday, G. Arutyunov and A. A. Tseytlin, "On integrability of classical superstrings in $\operatorname{AdS}(5) x S^{* *}$," JHEP 0507 (2005) 002 [arXiv:hep-th/0502240].

[31] A. Das, J. Maharana, A. Melikyan and M. Sato, "The algebra of transition matrices for the $A d S(5) x S^{* *} 5$ superstring," JHEP 0412 (2004) 055 [arXiv:hep-th/0411200].

[32] N. Beisert, V. A. Kazakov and K. Sakai, "Algebraic curve for the SO(6) sector of AdS/CFT," Commun. Math. Phys. 263 (2006) 611 [arXiv:hep-th/0410253].

[33] V. A. Kazakov and K. Zarembo, "Classical / quantum integrability in non-compact sector of AdS/CFT," JHEP 0410 (2004) 060 [arXiv:hep-th/0410105].

[34] N. Berkovits, "BRST cohomology and nonlocal conserved charges," JHEP 0502 (2005) 060 [arXiv:hep-th/0409159].

[35] M. Hatsuda and K. Yoshida, "Classical integrability and super Yangian of superstring on $\operatorname{AdS}(5) x S^{* *} 5$," Adv. Theor. Math. Phys. 9 (2005) 703 [arXiv:hep-th/0407044].

[36] V. A. Kazakov, A. Marshakov, J. A. Minahan and K. Zarembo, "Classical / quantum integrability in AdS/CFT," JHEP 0405 (2004) 024 [arXiv:hep-th/0402207].

[37] L. F. Alday, "Non-local charges on AdS(5) x $S^{* * 5}$ and pp-waves," JHEP 0312 (2003) 033 [arXiv:hep-th/0310146].

[38] B. C. Vallilo, "Flat currents in the classical AdS(5) $x S^{* *} 5$ pure spinor superstring," JHEP 0403 (2004) 037 [arXiv:hep-th/0307018]. 
[39] J. A. Minahan, "A Brief Introduction To The Bethe Ansatz In N=4 Super-Yang-Mills," J. Phys. A 39 (2006) 12657.

[40] N. Beisert, "The dilatation operator of $N=4$ super Yang-Mills theory and integrability," Phys. Rept. 405 (2005) 1 [arXiv:hep-th/0407277].

[41] J. Plefka, "Spinning strings and integrable spin chains in the AdS/CFT correspondence," arXiv:hep-th/0507136.

[42] A. A. Tseytlin, "Semiclassical strings and AdS/CFT," arXiv:hep-th/0409296.

[43] K. Zarembo, "Semiclassical Bethe ansatz and AdS/CFT," Comptes Rendus Physique 5 (2004) 1081 [Fortsch. Phys. 53 (2005) 647] [arXiv:hep-th/0411191].

[44] I. J. Swanson, "Superstring holography and integrability in $A d S(5) x S^{* * 5}$," arXiv:hep-th/0505028.

[45] A. A. Tseytlin, "Spinning strings and AdS/CFT duality," arXiv:hep-th/0311139.

[46] O. Aharony, "A brief review of 'little string theories'," Class. Quant. Grav. 17 (2000) 929 [arXiv:hep-th/9911147].

[47] D. Kutasov, "Introduction To Little String Theory," Prepared for ICTP Spring School on Superstrings and Related Matters, Trieste, Italy, 2-10 Apr 2001

[48] M. Henneaux and C. Teitelboim, "Quantization of gauge systems," Princeton, USA: Univ. Pr. (1992) $520 \mathrm{p}$

[49] J. Govaerts, "Hamiltonian Quantization And Constrained Dynamics," Leuven, Belgium: Univ. Pr. (1991) 371 p. (Leuven notes in mathematical and theoretical physics, B4)

[50] J. Govaerts, "The quantum geometer's universe: Particles, interactions and topology," arXiv:hep-th/0207276. 